www.nature.com/jhg

\title{
GST Theta null genotype is associated with an increased risk for ulcerative colitis: a case-control study and meta-analysis of GST Mu and GST Theta polymorphisms in inflammatory bowel disease
}

\author{
Mark MTJ Broekman, Caro Bos, René HM te Morsche, Frank Hoentjen, Hennie MJ Roelofs, \\ Wilbert HM Peters, Geert JA Wanten and Dirk J de Jong
}

Glutathione $S$-transferases (GSTs) are important in the detoxification of many compounds, including reactive oxygen species. Polymorphisms in GSTs resulting in a decreased enzyme activity might enhance the risk for inflammatory bowel disease by eliciting a state of oxidative stress. Previous case-control studies showed divergent results and were frequently limited in sample size; therefore we conducted a meta-analysis including results from our case-control study. For the case-control study, we genotyped 552 patients with Crohn's disease (CD), 223 patients with ulcerative colitis (UC) and 972 healthy controls by PCR for functional deletions in GST Mu and GST Theta. Both were not analyzed in recent genome-wide association studies. For the meta-analysis, PubMed, EMBASE and Web of Science were searched. In this meta-analysis, we show an enhanced susceptibility for UC in individuals with the GSTT1 null genotype (odds ratio (OR) $2.27,95 \%$ confidence interval (CI) 1.31 3.92). In our case-control study, a reduced risk for CD was seen with the GSTT1 null genotype (OR $0.58,95 \% \mathrm{Cl} 0.43-0.77)$; however, pooled analysis showed an OR of $1.67,95 \% \mathrm{Cl} 0.81-3.45$. In this meta-analysis, we showed an increased risk for UC in individuals with the GSTT1null genotype.

Journal of Human Genetics (2014) 59, 575-580; doi:10.1038/jhg.2014.77; published online 4 September 2014

\section{INTRODUCTION}

Genetic factors become more and more recognized as contributing factors in the development of inflammatory bowel disease (IBD). Recent genome-wide association studies (GWASs) generated a large set of candidate genes for Crohn's disease (CD), ulcerative colitis (UC) and for IBD in general. ${ }^{1}$

Glutathione $S$-transferases (GSTs) are a family of enzymes that have an essential role within cells, including the conjugation and detoxification of toxic or carcinogenic compounds, such as reactive oxygen species (ROS). ${ }^{2}$ Polymorphisms in GSTs can lead to a decreased enzyme function, and an inadequate detoxification of ROS might modulate the susceptibility for IBD. ${ }^{3}$ Biopsies of colonic mucosa of IBD patients showed an increased ROS production compared with healthy controls. ${ }^{4}$ A reduced enzyme function of GSTs and therewith impaired scavenging of ROS can contribute to a state of oxidative stress, which can trigger the onset of IBD. ${ }^{5,6}$

Human GSTs can be divided in four main classes, GST Alpha (GSTA), GST Mu (GSTM1), GST Pi (GSTP1) and GST Theta (GSTT1). For GSTP1, several polymorphisms have been described, with GSTP1 105 (rs1695) and GSTP1 114 (rs1138272) considered as the most important, because these GSTP1 polymorphisms result in a reduced enzyme function. ${ }^{7}$ Both GSTP1 105 and GSTP1 114 were not found to be associated with UC or CD in a GWAS. ${ }^{1}$ For GSTM1, three alleles have been described of which one, GSTM ${ }^{*} 0$, is characterized by a gene deletion, leading to a non-functional protein. Homozygous presence of the GSTM1 ${ }^{*} 0$ allele (GSTM1null) results in no enzyme activity and is found in approximately $50 \%$ of Caucasians and Asians. ${ }^{2,8}$ The same applies to GSTT1, where a gene deletion $\left(G S T T 1^{\star} 0\right.$ allele) is responsible for a failed protein synthesis. The homozygous $G S T T 1^{\star} 0$ genotype, GSTT1null, which occurs in approximately $10-20 \%$ of Caucasians and $16-64 \%$ of Asians, leads to the absence of GSTT1 enzyme activity. ${ }^{2,8}$ The gene deletions of GSTM $1^{\star} 0$ or $G S T T 1^{*} 0$ were not analyzed in GWASs, ${ }^{1}$ so evidence is limited to case-control studies. In these studies, an association between $G S T M 1^{*} 0, G S T T 1^{*} 0$ or a combination of these polymorphisms and susceptibility for CD or UC was found. However, it has to be stressed that some studies had relatively low patient numbers, and overall results remain inconsistent. ${ }^{9-12}$ Therefore we conducted a large 
case-control study and included our results in a meta-analysis of studies addressing the susceptibility for $\mathrm{CD}$ and $\mathrm{UC}$ with respect to GSTM1 and GSTT1 null polymorphisms.

\section{MATERIALS AND METHODS}

\section{Study design and ethics}

For the case-control study, IBD patients were recruited at the outpatient clinic of the Radboud University Medical Center, Nijmegen, the Netherlands. Healthy controls were recruited from the Nijmegen area, by advertisement in local papers, as described earlier. ${ }^{13}$ Diagnosis of IBD was based on accepted clinical, endoscopic, radiological and histological findings. ${ }^{14}$ The investigations were approved by the Medical Ethical Review Committee of the Radboud University Nijmegen Medical Center under the protocol number CWOM-nr 9804-0100.

\section{DNA isolation and genotyping}

Peripheral blood samples of IBD patients and healthy controls were collected and genomic DNA was isolated with the ROCHE High Pure PCR template Preparation Kit (Roche Applied Science, Mannheim, Germany). DNA was stored at $4{ }^{\circ} \mathrm{C}$ until further use. Polymorphisms in GSTM1 and GSTT1 were analyzed using PCR as described previously. ${ }^{15}$ The primers $5^{\prime}$-CTGGATTGTA GCAGATCATGC- $3^{\prime} / 5^{\prime}$-CTCCTGATTATGACAGAAGCC- $3^{\prime}$ and $5^{\prime}$-TCACCGG ATCATGGCCAGCA-3' $/ 5^{\prime}$-TTCCTTACTGGTCCTCACATCTC- $3^{\prime}$ were used for GSTM1 and GSTT1, respectively. To detect GSTM1 and GSTT1 genotypes, melt curve analysis was applied using the Bio-Rad Precision Melt Analysis Software version 1.0 (Bio-Rad, Hercules, CA, USA) and in this way homozygous and heterozygous most common genotypes could be distinguished from homozygous $\mathrm{GSTM1}^{*} 0$ (null) or $\mathrm{GSTT1}^{*} 0$ (null) genotypes.

\section{Data search}

The meta-analysis was conducted according to PRISMA guidelines. Articles for the meta-analyses were retrieved by searching PubMed, EMBASE and Web of Science on 13 October 2013 with the following search terms: GST, glutathione $S$-transferase, detoxification enzymes, inflammatory bowel disease, ulcerative colitis, and Crohn's disease. Case-control studies examining the association of GST polymorphisms in patients with IBD in relation to healthy controls were included, provided that absolute numbers of genotypes of patients and controls were given. Studies were excluded when no full text was available or written in a language other than English, German or Dutch. In case of overlapping data, the study with the largest number of cases was included. The reference lists of included articles were screened for potential eligible studies. Two researchers (MB and RM) independently extracted raw data of the genotype distributions of GSTM1, GSTT1 and the combination of GSTM1 with GSTT1 from the included studies, and in case of inconsistency, results were discussed until consensus was reached.

\section{Statistical analysis}

For the case-control study, baseline continuous variables were compared by independent $t$-test. Categorical data between patients and healthy controls were analyzed with the chi-squared test. Genotypes with predicted high enzyme activity were used as reference to calculate odds ratios (OR) and 95\% confidence intervals (95\% CI) with logistic regression. The combination GSTM1null and GSTTInull was compared with all other genotypes. Analyses were conducted with the SPSS for windows version 20.0 (SPSS Inc., Chigaco, IL, USA).

For the meta-analyses a random effect model was used to calculate pooled ORs from studies, including results of our case-control study. ORs with $95 \%$ CI were calculated with the Mantel-Haenszel method in Review Manager (RevMan) version 5.2. (The Nordic Cochrane Centre, The Cochrane Collaboration, Copenhagen, Denmark). The $P$-values of significance of the ORs are depicted in the bottom line of the corresponding forest plots. Finally, heterogeneity was assessed with the $I^{2}$ test, and publication bias was estimated with a funnel plot.

\section{RESULTS}

\section{Case-control study}

For the case-control study, we included 552 patients with CD (mean age 44.4 years, s.d. \pm 13.8 ), 233 patients with UC (mean age 45.8 years, s.d. \pm 13.7 ) and 972 healthy controls (mean age 47.2 years, s.d. \pm 16.6 ). Table 1 shows the GST genotype distribution in patients with $\mathrm{CD}$ and UC in comparison with healthy controls. With the homozygous + heterozygous most common genotypes set as reference, the GSTM1null genotype was not associated with a significant increased susceptibility for CD or UC. The GSTT1null genotype was more often present in healthy controls compared with patients with CD (OR 0.58; 95\% CI 0.43-0.77). Although in UC patients the GSTT1null genotype was more frequently seen, difference was not significant (OR 1.14, 95\% CI 0.81-1.61). The combination of GSTM1null and GSTT1null genotypes was significantly seen more in healthy controls compared with patients with CD (OR 0.44, 95\% CI 0.29-0.66). The association with the GSTT1null genotype and CD and the combination of GSTT1null and GSTM1null had a P-value of $<0.001$. This was still significant after correction for multiple testing (Bonferroni's correction).

\section{Study characteristics of the meta-analyses}

In total, 251 articles were identified. After removing duplicates and screening for title and abstract, 11 articles remained. ${ }^{9-12,16-22}$ Three studies $^{20-22}$ were excluded because of Chinese language. One study was excluded ${ }^{18}$ because of overlapping data with the results of our case-control study. In the study of Karban et al., ${ }^{10}$ the results of Jewish, Arab moslems and Druze were merged. No studies had to be excluded because of no full text availability. Furthermore, all studies used genotyping methods that can detect the null genotype with equivalent accuracy, except one study that used a genotype-validated assay based on the GSTMu activity to identify patients with the null genotype.

\section{Results of the meta-analyses}

No increased susceptibility was found for CD with the GSTM1null (Figure 1) or GSTT1null genotype (Figure 2; OR 1.08, 95\% CI 0.84

Table 1 GST genotype distributions and OR with $95 \% \mathrm{Cl}$ of patients with $\mathrm{CD}$ and UC compared with controls in our case-control study

\begin{tabular}{|c|c|c|c|c|c|c|c|}
\hline GST & Genotype & Predicted enzyme activity & Controls (\%) & $C D(\%)$ & OR $(95 \% \mathrm{Cl})$ & UC (\%) & OR $(95 \% \mathrm{Cl})$ \\
\hline \multirow[t]{2}{*}{ GSTM1 } & $* 1 * 1 / * 1 * 0$ & High-intermediate & $442(45.5)$ & $263(47.6)$ & Reference & $116(49.8)$ & Reference \\
\hline & *0/*0 & Absent & $530(54.5)$ & $289(52.4)$ & $0.92(0.74-1.13)$ & $117(50.2)$ & $0.84(0.63-1.12)$ \\
\hline \multirow[t]{2}{*}{ GSTT1 } & $* 1 * 1 /{ }^{*} 1 * 0$ & High-intermediate & 769 (79.1) & $479(86.8)$ & Reference & 179 (76.8) & Reference \\
\hline & ${ }^{*} 0 /{ }^{*} 0$ & Absent & 203 (20.9) & $73(13.2)$ & $0.58(0.43-0.77)^{*}$ & $54(23.2)$ & $1.14(0.81-1.61)$ \\
\hline \multirow[t]{2}{*}{ GSTM1 and GSTT1 } & ${ }^{*} 1 * 1 / * 1 * 0$ and $* 1 * 1 / * 1 * 0$ & High-intermediate & $850(87.4)$ & $490(94)$ & Reference & 209 (89.7) & Reference \\
\hline & ${ }^{*} 0 /{ }^{*} 0$ and ${ }^{*} 0 /{ }^{*} 0$ & Absent & $122(12.6)$ & $31(6.0)$ & $0.44(0.29-0.66)^{*}$ & 24 (10.3) & $0.80(0.50-1.27)$ \\
\hline
\end{tabular}

Abbreviations: CD, Crohn's disease; Cl, confidence interval; GST, glutathione S-transferase; GSTM1, GST Mu; GSTT1, GST Theta; OR, odds ratio; UC, ulcerative colitis. ORs were calculated with the genotypes with high-intermediate enzyme activity set as reference. ${ }^{*} P<0.001$ 


\begin{tabular}{lrrrrr} 
& \multicolumn{3}{c}{ Crohn's disease } & \multicolumn{4}{c}{ Healthy controls } \\
Study or Subgroup & Events & Total & Events & Total & Weight \\
\hline Duncan 1995 & 68 & 110 & 203 & 373 & $14.8 \%$ \\
Hertevig 1995 & 65 & 109 & 219 & 449 & $15.1 \%$ \\
Mittal 2007 & 9 & 20 & 49 & 164 & $5.7 \%$ \\
Ernst 2010 & 215 & 386 & 417 & 791 & $21.2 \%$ \\
Karban 2011 & 208 & 436 & 300 & 528 & $20.8 \%$ \\
Broekman 2014 & 289 & 552 & 530 & 972 & $22.3 \%$ \\
& & & & & \\
Total (95\% Cl) & & 1613 & & 3277 & $100.0 \%$ \\
Total events & 854 & & 1718 & \\
Heterogeneity: Tau $^{2}=0.06 ;$ Chi $^{2}=17.31, \mathrm{df}=5(\mathrm{P}=0.004) ; I^{2}=71 \%$ \\
Test for overall effect: $Z=0.61(\mathrm{P}=0.54)$
\end{tabular}

Odds Ratio

M-H, Random, 95\% Cl Year

$1.36[0.88,2.10] 1995$

$1.55[1.01,2.37] 1995$

$1.92[0.75,4.93] 2007$

$1.13[0.88,1.44] 2010$

$0.69[0.54,0.89] 2011$

$0.92[0.74,1.13] 2014$

$1.08[0.84,1.39]$

Figure 1 Forest plot of the association of the GSTM1null genotype with Crohn's disease (CD). Effect of GSTM1null on the risk of CD (odds ratios (ORs) and the $95 \%$ confidence intervals $(\mathrm{Cls})$ ) for each study is shown. The overall OR is $1.08(95 \% \mathrm{Cl} 0.84-1.39)$. GSTM1null, homozygous presence of the GSTM $1 * 0$ allele.

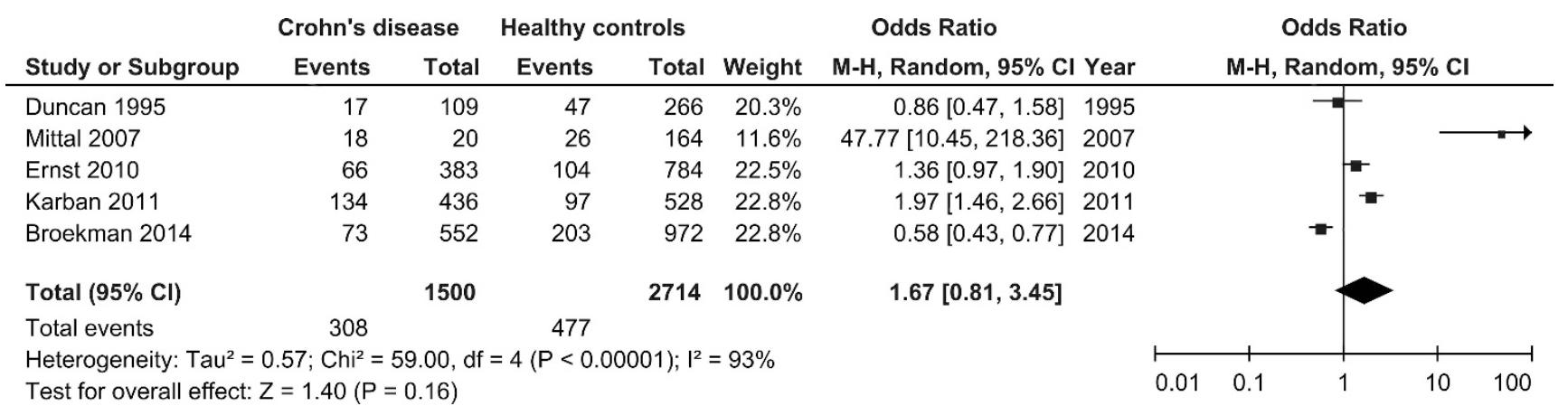

Figure 2 Forest plot of the association of the GSTT1null genotype with Crohn's disease (CD). Effect of the GSTT1null genotype on the risk of CD (odds ratios (ORs) and the $95 \%$ confidence intervals (Cls)) for each study is shown. The overall OR is 1.67 (95\% Cl 0.81-3.45). GSTT1null, homozygous presence of the $G S T T 1^{*} O$ allele.

\begin{tabular}{|c|c|c|c|c|c|c|c|c|c|c|}
\hline \multirow[b]{2}{*}{ Study or Subgroup } & \multicolumn{2}{|c|}{ Crohn's disease } & \multicolumn{2}{|c|}{ Healthy controls } & \multicolumn{3}{|c|}{ Odds Ratio } & \multirow{2}{*}{\multicolumn{3}{|c|}{$\begin{array}{c}\text { Odds Ratio } \\
\text { M-H, Random, } 95 \% \mathrm{Cl}\end{array}$}} \\
\hline & Events & Total & Events & Total & Weight & M-H, Random, $95 \% \mathrm{Cl}$ & Year & & & \\
\hline Mittal 2007 & 4 & 20 & 8 & 164 & $26.0 \%$ & $4.88[1.32,17.99]$ & 2007 & & & $\longrightarrow$ \\
\hline Ernst 2010 & 36 & 384 & 50 & 787 & $36.8 \%$ & $1.52[0.98,2.38]$ & 2010 & & & -1 \\
\hline Broekman 2014 & 31 & 521 & 122 & 972 & $37.1 \%$ & $0.44[0.29,0.66]$ & 2014 & & $=-$ & \\
\hline Total $(95 \% \mathrm{Cl})$ & & 925 & & 1923 & $100.0 \%$ & $1.30[0.41,4.14]$ & & & & \\
\hline Total events & 71 & & 180 & & & & & & & \\
\hline \multicolumn{8}{|c|}{ Heterogeneity: $\mathrm{Tau}^{2}=0.90 ; \mathrm{Chi}^{2}=23.61, \mathrm{df}=2(P<0.00001) ; \mathrm{I}^{2}=92 \%$} & $1 \frac{1}{1}$ & 0.5 & 5 \\
\hline
\end{tabular}

Figure 3 Association of the combination of GSTM1null and GSTT1null genotypes with Crohn's disease (CD). Risk of CD (odds ratios (ORs) and the 95\% confidence intervals (Cls)) with both the GSTM1 null and GSTT1null genotype versus all other variants is shown for each study. The overall OR is 1.30 ( $95 \%$ CI 0.41-4.14). GSTM1null, homozygous presence of the GSTM1*O allele; GSTT1 null, homozygous presence of the GSTT1*O allele.

1.39 and $1.67,95 \%$ CI $0.81-3.45$, respectively). Also the combination of both did not influence disease susceptibility (OR 1.30, 95\% CI 0.41-4.14; Figure 3). Pooled analysis revealed an increased risk for UC with the GSTT1null genotype (OR 2.27, 95\% CI 1.31-3.92; Figure 4), still significant after correction for multiple testing $(P=0.003)$. For GSTM1null (OR 1.35, 95\% CI 0.89-2.03) and the combination of GSTT1null and GSTM1null (OR 1.99, 95\% CI 0.65-6.12), no significant influence on disease susceptibility was seen (see Figures 5 and 6).
Publication bias

The funnel plot from the pooled analysis of GSTT1 in UC, the only meta-analysis showing a significant effect, is depicted in Figure 7. This meta-analysis included seven studies, and with the exception of one outlier, the shape of the plot appears symmetrical.

\section{DISCUSSION}

The results of the current meta-analyses show a significantly increased risk for UC, but not for CD, when bearing the GSTT1null genotype. 


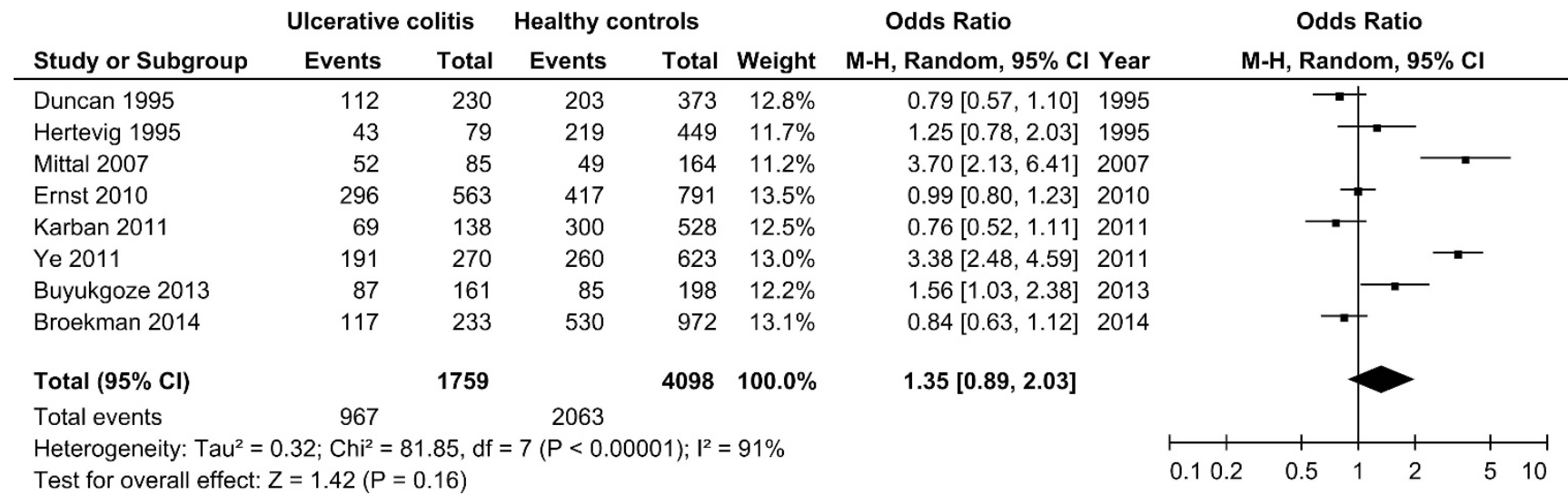

Figure 4 Forest plot of the association of the GSTTInull genotype with ulcerative colitis (UC). Effect of the GSTTInull genotype on the risk of UC (odds ratios (ORs) and the 95\% confidence intervals (Cls)) for each study is shown. The overall OR is 2.27 (95\% Cl 1.31-3.92). GSTT1null, homozygous presence of the $G S T T 1{ }^{*} 0$ allele.

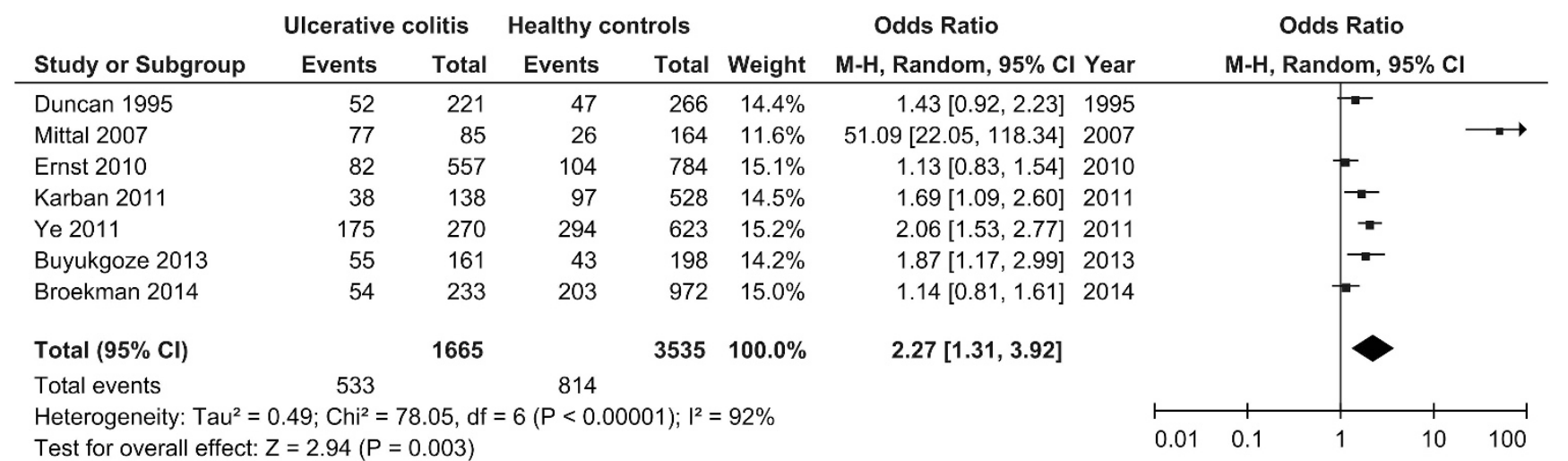

Figure 5 Forest plot of the association of the GSTM1null genotype with ulcerative colitis (UC). Effect of the GSTM1null genotype on the risk of UC (odds ratios (ORs) and the $95 \%$ confidence intervals (Cls)) for each study is shown. The overall OR is 1.35 (95\% Cl 0.89-2.03). GSTM1null, homozygous presence of the $G S T M 1{ }^{*} 0$ allele.

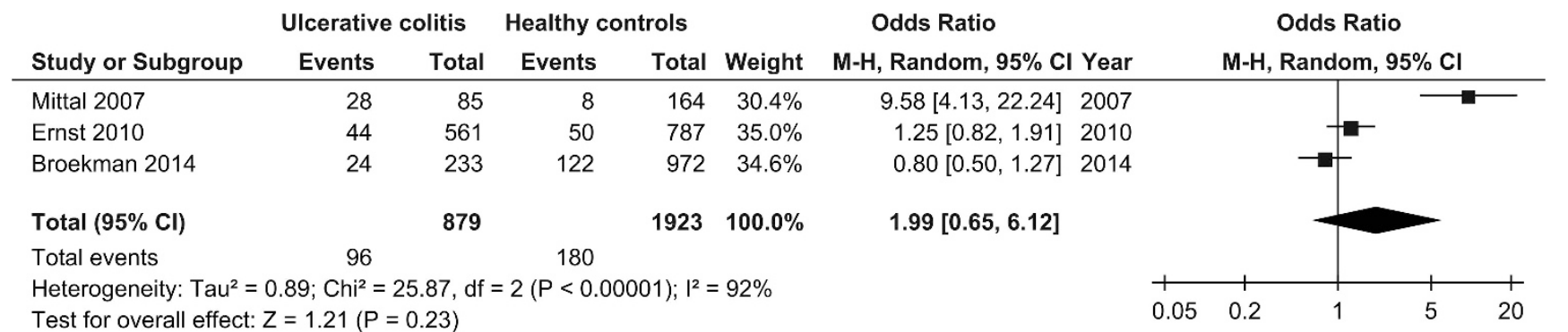

Figure 6 Association of the combination of GSTT1null and GSTM1null genotypes with ulcerative colitis (UC). Risk of UC (odds ratios (ORs) and $95 \%$ confidence intervals (Cls)) with both the GSTM1null and GSTT1 null genotype versus all other variants is shown for each study. The overall OR is 1.99 (95\% $\mathrm{Cl}$ 0.65-6.12). GSTM1null, homozygous presence of the GSTM1*O allele; GSTT1null, homozygous presence of the GSTT1 *0 allele.

The GSTM1null genotype was not associated with CD or UC. It is important to mention that the GSTM1null and GSTT1null polymorphisms were not analyzed in GWASs, ${ }^{1}$ because these polymorphisms consist of large deletions rather than singlenucleotide substitutions. With pooling data from case-control studies, supplemented with our data, overall IBD patient numbers exceed 3200 , which makes this meta-analyses the largest study so far.
The mechanism that absence of GSTT1 enzyme activity in GSTT1null individuals contributes to the risk for UC might be explained by the important role of this enzyme in the detoxification of ROS, ${ }^{23}$ which may provide a trigger in the etiology of IBD. ${ }^{24,25}$ Also, in other inflammatory-driven diseases, such as asthma or type 2 diabetes mellitus, an increased susceptibility was found with the GSTT1null genotype. ${ }^{26,27}$ In this meta-analyses, we showed that the 


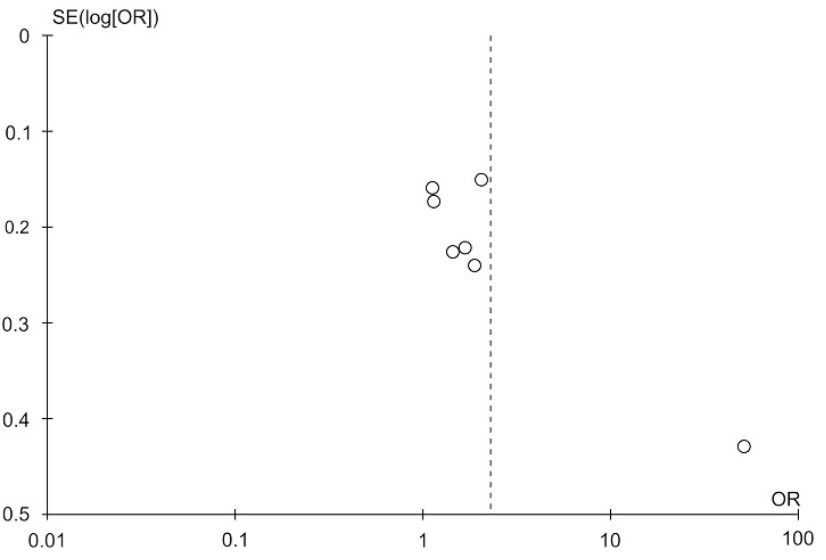

Figure 7 Funnel plot for the meta-analysis of the GSTTInull genotype in ulcerative colitis to detect potential publication bias.

GSTT1null genotype is associated with UC but not with CD. This finding is not unique as 53 of the 163 loci associated with IBD were disease specific. ${ }^{1}$

Although the studies conducted in Asia showed a larger association between the GSTT1null genotype and UC than European studies, all studies revealed an increased risk with only variation in the magnitude. When the studies of Mittal et al. ${ }^{19}$ (OR 51.09, 95\% CI 22.05-118.34) and Ye et al. ${ }^{9}$ (OR 2.06, 95\% CI 1.53-2.77) were excluded from the pooled analysis, leaving only Caucasians studies, the GSTT1null genotype was still significantly seen more in UC patients (OR 1.36, 95\% CI 1.11-1.65). The increased, though not significant susceptibility, for CD with the GSTT1null genotype is, to a large extent, caused by the study of Mittal et al. ${ }^{19}$ with an OR of 47.8 (95\% CI 10.5-218.4). The likely cause of this large OR is the low number of patients $(n=20)$ with CD included, which is reflected by the broad CI. When this study is excluded from the meta-analysis, the OR is 1.08 (95\% CI 0.59-2.00). Furthermore, in the forest plot of GSTT1 in CD, a large difference was seen between the study of Ernst et al. ${ }^{11}$ and our results, despite only a small geographical difference. This might be caused by a relatively low number of healthy controls with the GSTT1null genotype in the Danish study compared with our study ( $13.2 \%$ vs $20.8 \%$, respectively), where $19.7 \%$ is the average for this area. ${ }^{8}$

Also, with the GSTM1null genotype, both the two studies conducted in Asia (Mittal et al. ${ }^{19}$ and Ye et al. ${ }^{9}$ ) revealed the highest association with IBD. However, overall no significant influence was found. It might be that the loss of function of the GSTM1 enzyme is compensated by other GST Mu classes, such as GSTM2, ${ }^{28}$ this also explains why the combination of both GSTM1null and GSTT1null did not have a synergistic effect on disease susceptibility. Another explanation could be that the expression of GSTM1 in the bowel is limited and therefore does not contribute in the pathogenesis of IBD. ${ }^{2}$

The difference between the studies conducted in Asia and Europe support the recent insights in differences in genetic variants associated with IBD between the Caucasian and Asian race. ${ }^{29,30} \mathrm{Ng}$ et al. ${ }^{29}$ showed that genetic variants of NOD2 frequently seen in Caucasian IBD patients were rare in the Asian IBD population and vice versa. Therefore it can be hypothesized that for Asians both GSTT1null and GSTM1null may be involved in the etiology of UC, whereas in Caucasians only the GSTT1null genotype is associated with UC. This distinction might result from differences in life style, environmental factors and the microbiome, requiring a different utilization of GST subclasses. Ideally, subgroup analyses for different races are included; however, absolute numbers of Asian IBD patients are insufficient for a reliable estimation. This accentuates the need for larger studies in the Asian IBD population exploring GSTM1null as well as GSTT1null genotypes.

Single-nucleotide polymorphisms (SNPs) in the neighbor of the deletion polymorphism may be in linkage disequilibrium and subsequently might be used as a tag SNP. However, this method is limited by the inter-population differences in the degree of linkage disequilibrium. Most SNPs in linkage disequilibrium with GSTM1 and GSTT1 ${ }^{31}$ were not included in the most recent GWAS. ${ }^{1}$ This can partly be explained by the fact that some of these tag SNPs such as rs407257 lie in the deletion area.

As previous studies with comparable or smaller sample sizes were able to show significant associations, we assumed to have sufficient power with the patient numbers included in our case-control study. In the present meta-analysis, heterogeneity between studies was observed, which besides differences in race could have been originated from differences in study design. For example, patient enrolment in academic hospitals could be confounded by more severe disease phenotypes included in academic hospitals. Also, the selection of healthy controls might have an impact and could be a possible cause for heterogeneity. Considering these potential differences, we used a random-effect model for computing pooled ORs, with the knowledge that in case of low heterogeneity results of a random model will approach values of a fixed model, but preventing poorly substantiated significant outcomes. ${ }^{32}$ The funnel plot of GSTT1null in UC contains one outlier (the study of Mittal et al. ${ }^{19}$ with an OR of 51.09), though, as mentioned above, with exclusion of this study still a significant effect of GSTT1null and UC was observed. It has to be mentioned that the number of studies published on this topic is limited, which makes correct interpretation of the funnel plot difficult. Therefore influence of publication bias should be considered, as studies showing a significant influence of specific polymorphisms might be published more often. ${ }^{33}$ Furthermore, the PCR techniques used in our casecontrol study, and the other included studies, can only detect homozygous (null) genotypes of the $G S T M 1^{*} 0$ and $G S T T 1^{*} 0$ alleles, failing to detect heterozygous individuals. One study measured GSTM1 enzyme activity instead of the GSTM1null genotype. ${ }^{12}$ We decided to include this study in the meta-analyses as this method is validated against the GSTM1 genotype and their findings showed that $50 \%$ of controls had no GSTM1 activity, which is in line with the GSTM1null genotype frequency in that geographical area., ${ }^{8,34}$ In addition, this method also lacks the capability to identify GSTM1null heterozygotes

In conclusion, in this meta-analysis we show that the GSTT1null genotype is associated with an increased the risk for UC but not for CD.

\section{CONFLICT OF INTEREST}

The authors declare no conflict of interest.

1 Jostins, L., Ripke, S., Weersma, R. K., Duerr, R. H., McGovern, D. P., Hui, K. Y. et al. Host-microbe interactions have shaped the genetic architecture of inflammatory bowel disease. Nature 491, 119-124 (2012).

2 Hayes, J. D. \& Strange, R. C. Glutathione S-transferase polymorphisms and their biological consequences. Pharmacology 61, 154-166 (2000).

3 Conner, E. M., Brand, S. J., Davis, J. M., Kang, D. Y. \& Grisham, M. B. Role of reactive metabolites of oxygen and nitrogen in inflammatory bowel disease: toxins, mediators, and modulators of gene expression. Inflamm. Bowel. Dis. 2, 133-147 (1996). 
4 Simmonds, N. J., Allen, R. E., Stevens, T. R., Van Someren, R. N., Blake, D. R. \& Rampton, D. S. Chemiluminescence assay of mucosal reactive oxygen metabolites in inflammatory bowel disease. Gastroenterology 103, 186-196 (1992).

5 Pavlick, K. P., Laroux, F. S., Fuseler, J., Wolf, R. E., Gray, L., Hoffman, J. et al. Role of reactive metabolites of oxygen and nitrogen in inflammatory bowel disease. Free Radic Biol. Med. 33, 311-322 (2002)

6 Alzoghaibi, M. A. Concepts of oxidative stress and antioxidant defense in Crohn's disease. World J. Gastroenterol. 19, 6540-6547 (2013).

7 Watson, M. A., Stewart, R. K., Smith, G. B., Massey, T. E. \& Bell, D. A. Human glutathione S-transferase $\mathrm{P} 1$ polymorphisms: relationship to lung tissue enzyme activity and population frequency distribution. Carcinogenesis 19, 275-280 (1998).

8 Garte, S., Gaspari, L., Alexandrie, A. K., Ambrosone, C., Autrup, H., Autrup, J. L. et al. Metabolic gene polymorphism frequencies in control populations. Cancer Epidemiol. Biomarkers Prev. 10, 1239-1248 (2001).

9 Ye, X., Jiang, Y., Wang, H., Chen, L., Yuan, S. \& Xia, B. Genetic polymorphisms of glutathione S-transferases are associated with ulcerative colitis in central China. Cell Biochem. Biophys. 60, 323-328 (2011).

10 Karban, A., Krivoy, N., Elkin, H., Adler, L., Chowers, Y., Eliakim, R. et al. Non-Jewish Israeli IBD patients have significantly higher glutathione S-transferase GSTT1-null frequency. Dig. Dis. Sci. 56, 2081-2087 (2011).

11 Ernst, A., Andersen, V., Ostergaard, M., Jacobsen, B. A., Dagiliene, E., Pedersen, I. S. et al. Genetic variants of glutathione S-transferases mu, theta, and pi display no susceptibility to inflammatory bowel disease in the Danish population. Scand. J. Gastroenterol. 45, 1068-1075 (2010).

12 Hertervig, E., Nilsson, A. \& Seidegard, J. The expression of glutathione transferase mu in patients with inflammatory bowel disease. Scand. J. Gastroenterol. 29, 729-735 (1994).

13 Kristinsson, J. O., van Westerveld, P., te Morsche, R. H., Roelofs, H. M., Wobbes, T., Witteman, B. J. et al. Cyclooxygenase-2 polymorphisms and the risk of esophagea adeno- or squamous cell carcinoma. World J. Gastroenterol. 15, 3493-3497 (2009).

14 Podolsky, D. K. Inflammatory bowel disease. N. Engl. J. Med. 347, 417-429 (2002).

15 Dura, P., Salomon, J., Te Morsche, R. H., Roelofs, H. M., Kristinsson, J. O., Wobbes, T. et al. No role for glutathione S-transferase genotypes in Caucasian esophagea squamous cell or adenocarcinoma etiology: an European case-control study. BMC Gastroenterol. 13, 97 (2013).

16 Duncan, H., Swan, C., Green, J., Jones, P., Brannigan, K., Alldersea, J. et al. Susceptibility to ulcerative colitis and Crohn's disease: interactions between glutathione S-transferase GSTM1 and GSTT1 genotypes. Clin. Chim. Acta 240, 53-61 (1995).

17 Buyukgoze, O., Osmanoglu, N., Arslan, S. \& Sen, A. Association of the CYP1A1*2A, GSTT1 null, GSTM1 null, $m E P H X * 3$, and XRCC1-399 genetic polymorphisms with ulcerative colitis. Int. J. Colorectal Dis. 28, 593-595 (2013).

18 de jong, D. J., van der Logt, E. M., van Schaik, A., Roelofs, H. M., Peters, W. H. \& Naber, T. H. Genetic polymorphisms in biotransformation enzymes in Crohn's disease: association with microsomal epoxide hydrolase. Gut 52, 547-551 (2003).

19 Mittal, R. D., Manchanda, P. K., Bid, H. K. \& Ghoshal, U. C. Analysis of polymorphisms of tumor necrosis factor-alpha and polymorphic xenobiotic metabolizing enzymes in inflammatory bowel disease: study from northern India. J. Gastroenterol. Hepatol. 22, 920-924 (2007)

20 Xia, X., Wang, W. Jiang, Y. Lin, L. \& Chen, H. Relationship between gene polymorphism of glutathione S-transferase M1 and susceptibility to ulcerative colitis. [Chinese]. Chin. J Gastroenterol. 12, 295-297 (2007).

21 Wang, W. X., Xia, X. P., Jiang, Y., Lin, L. M., Zhang, D. L. \& Cao, S. G. Association between genetic polymorphism of glutathione S-Transferase T1 and the susceptibility to ulcerative colitis. [Chinese]. World Chin. J. Digestol. 15, 533-536 (2007).

$22 \mathrm{Wu}$, H., Zheng, B., Wang, J. Z., Pei, J. H., Jiang, L. N. \& Xue, Z. X. Relationship between genetic polymorphisms of glutathione S-transferase genes and susceptibility to ulcerative colitis in Zhejiang Han population. [Chinese]. World Chin. J. Digestol. 18, 2780-2784 (2010)

23 Hayes, J. D. \& Pulford, D. J. The glutathione S-transferase supergene family: regulation of GST and the contribution of the isoenzymes to cancer chemoprotection and drug resistance. Crit. Rev. Biochem. Mol. Biol. 30, 445-600 (1995).

24 Iborra, M., Moret, I., Rausell, F., Bastida, G., Aguas, M., Cerrillo, E. et al. Role of oxidative stress and antioxidant enzymes in Crohn's disease. Biochem. Soc. Trans. 39, 1102-1106 (2011).

25 Simmonds, N. J. \& Rampton, D. S. Inflammatory bowel disease-a radical view. Gut 34 865-868 (1993).

26 Zhao, Y., Liu, S., Liu, Z., Ye, Y. \& Mao, M. Significant association between GSTT1 null genotype and risk of asthma during childhood in Caucasians. Mol. Biol. Rep. 40, 1973-1978 (2013).

27 Pinheiro, D. S., Rocha Filho, C. R., Mundim, C. A., Junior Pde, M., Ulhoa, C. J. Reis, A. A. et al. Evaluation of glutathione S-transferase GSTM1 and GSTT1 deletion polymorphisms on type-2 diabetes mellitus risk. PLoS One 8, e76262 (2013).

28 Bhattacharjee, P., Paul, S., Banerjee, M., Patra, D., Banerjee, P., Ghoshal, N. et al. Functional compensation of glutathione S-transferase M1 (GSTM1) null by another GST superfamily member, GSTM2. Sci. Rep. 3, 2704 (2013).

29 Ng, S. C., Tsoi, K. K., Kamm, M. A., Xia, B., Wu, J., Chan, F. K. et al. Genetics of inflammatory bowel disease in Asia: systematic review and meta-analysis. Inflamm. Bowel. Dis. 18, 1164-1176 (2012).

30 Yang, S. K., Hong, M., Zhao, W., Jung, Y., Baek, J., Tayebi, N. et al. Genome-wide association study of Crohn's disease in Koreans revealed three new susceptibility loci and common attributes of genetic susceptibility across ethnic populations. Gut 63 80-87 (2014).

31 McCarroll, S. A., Hadnott, T. N., Perry, G. H., Sabeti, P. C., Zody, M. C., Barrett, J. C. et al. Common deletion polymorphisms in the human genome. Nat. Genet. 38, 86-92 (2006).

32 Borenstein, M., Hedges, L. V., Higgins, J. P. T. \& Rothstein, H. R. A basic introduction to fixed-effect and random-effects models for meta-analysis. Res. Synth. Methods 1, 97-111 (2010).

33 Ioannidis, J. P., Trikalinos, T. A., Ntzani, E. E. \& Contopoulos-loannidis, D. G. Genetic associations in large versus small studies: an empirical assessment. Lancet $\mathbf{3 6 1}$ 567-571 (2003).

34 Seidegard, J., Vorachek, W. R., Pero, R. W. \& Pearson, W. R. Hereditary differences in the expression of the human glutathione transferase active on trans-stilbene oxide are due to a gene deletion. Proc. Natl Acad. Sci. USA 85, 7293-7297 (1988). 